\title{
Review of Papers Describing Neuroinformatics Software
}

\author{
Erik De Schutter • Giorgio A. Ascoli • David N. Kennedy
}

Published online: 20 November 2009

(C) Humana Press 2009

This and other specialized journals publish many papers that describe computer software, including programs for analyzing data (Duff et al. 2007; Srinivasan et al. 2007; Bagarinao et al. 2008; Condron 2008; Liu et al. 2008; Zhang et al. 2008; Glascher 2009; Goldberg et al. 2009; Gunay et al. 2009; Nowinski et al. 2009), assist in the acquisition or management of data (Brown et al. 2005; Bezgin et al. 2009), and for simulating computer models (Cannon et al. 2003; Ichikawa 2005; Versace et al. 2008; Koene et al. 2009). Like all papers submitted to the journal the manuscripts are thoroughly refereed by two or three independent reviewers for scientific quality and clarity of the exposition. Usually, however, the reviewers have to trust that the authors gave a fair description of the software. The situation is somewhat similar to the review of experimental papers, where the referees have to trust that the authors describe the experiments accurately and completely. In experimental science, it would be impractical to reproduce systematically the empirical claims. For computer software, in contrast, this limitation only reflects

E. De Schutter $(\bowtie)$

Computational Neuroscience Unit, Okinawa Institute of Science and Technology, Japan and Theoretical Neurobiology,

University of Antwerp,

Antwerp, Belgium

e-mail: erik@tnb.ua.ac.be

\section{G. A. Ascoli}

Center for Neural Informatics, Structures, \& Plasticity, and Molecular Neuroscience Departrment,

Krasnow Institute for Advanced Study, George Mason University, Fairfax, VA, USA

D. N. Kennedy

Division of Neuroinformatics, Department of Psychiatry,

University of Massachusetts Medical School,

Worcester, MA, USA an old-fashioned approach, stemming from a time when it was difficult to distribute code or executables, and when programs were often very platform-dependent. In this era of sharing of resources and data (Kennedy 2004) and of webbased software distribution (Gardner et al. 2008; Luo et al. 2009) it has become fairly easy to make the software itself also accessible to reviewers, opening possibilities for deeper review of software related papers. This opportunity is particularly meaningful for the field of neuroinformatics and its leading (and namesake) journal.

Over the last year our journal has been running a pilot program in which it asked reviewers of papers describing neuroinformatics programs to also evaluate the software itself. Often this required no extra work on the side of the authors because they were already making the software available for anonymous download. Otherwise we arranged that the action editor could make the software available to the anonymous reviewers. The results of this pilot program were interesting and encouraging. The most common problem, reported for several papers, was that the reviewers could simply not run the software due to installation or compilation problems. This is not entirely surprising: anybody who has distributed software that needs to be compiled or that depends on the presence of specific libraries (typically programs written in java or python) knows that installation problems are among the most frequent complaints of users. Whenever reviewers encountered this type of problem the response of authors was immediate and they clearly saw this feedback as beneficial for their software distribution effort. Other issues that arose during software review were processing speed and access to benchmarking results. From an editorial viewpoint this information was seminal in deciding whether the software was eligible for description as an Original Article or more suited for a News Item. 


\section{New Policy}

Based on the positive experience of the pilot program the editors of this journal have decided to make software review henceforward a requisite for acceptance of papers that primarily describe software products. We also provide clear instructions on the expected content of such manuscripts.

To be accepted as an Original Article the paper should either describe novel algorithms or a software implementation that clearly improves on other software packages with similar objectives. The manuscript should not be considered a technical manual for the software nor pure advertisement. The journal encourages the description of innovative programming solutions that may not be of interest to regular neuroscience journals. Sufficient attention should be given to validation issues and, whenever possible, comparative benchmarking of the software should be performed. The scientific usefulness of the new software compared to what is already available to scientists working in the domain of interest should be discussed.

Alternatively, authors can submit shorter papers describing the availability of relevant software under the News Item category. In this case, the main criterion will be novelty and general utility of the tool for the community, but less emphasis will be placed on algorithm and benchmarking. The usability of the program described in manuscripts submitted as News Items will also be peer-reviewed (by the editors and/or a referee) under this new policy.

Authors submitting manuscripts describing software packages (whether as Original Articles or News Items) are requested to ensure that the software as described in the paper is available for the review, either by sharing it publicly or by making specific arrangements with the editors of the journal (e.g. providing a password to a protected download site or by providing a file containing the software or installation package). They should also provide all the data, scripts, etc. necessary to generate the illustrations shown in the paper. The editors will invite a reviewer with the specific task of running the software and comparing it with the description in the manuscript. It is not the goal of the review to actually validate or benchmark the software itself, that is the responsibility of the authors. Nevertheless, acceptance by the journal will now confirm that the software operates as described.

We believe that this new policy is an important step in furthering the field while at the same time improving the quality of the journal. We expect that although it might become more difficult for authors to publish manuscripts describing software in Neuroinformatics, they will appreciate the implicit seal of quality that comes with successful publication. As we gain more experience with this deeper review of manuscripts submitted to the journal we will keep updating and improving our review policies.

\section{References}

Bagarinao, E., Matsuo, K., Nakai, T., \& Tanaka, Y. (2008). BAX: a toolbox for the dynamic analysis of functional MRI datasets. Neuroinformatics, 6, 109-115.

Bezgin, G., Reid, A. T., Schubert, D., \& Kötter, R. (2009). Matching spatial with ontological brain regions using Java tools for visualization, database access, and integrated data analysis. Neuroinformatics, 7, 7-22.

Brown, K. M., Donohue, D. E., D’Alessandro, G., \& Ascoli, G. A. (2005). A cross-platform freeware tool for digital reconstruction of neuronal arborizations from image stacks. Neuroinformatics, $3,343-60$.

Cannon, R. C., Hasselmo, M. E., \& Koene, R. A. (2003). From biophysics to behavior: Catacomb2 and the design of biologicallyplausible models for spatial navigation. Neuroinformatics, 1, 3-42.

Condron, B. G. (2008). A freeware java tool for spatial point analysis of neuronal structures. Neuroinformatics, 6, 57-61.

Duff, E. P., Cunnington, R., \& Egan, G. F. (2007). REX: response exploration for neuroimaging datasets. Neuroinformatics, 5, 223 234.

Gardner, D., et al. (2008). The neuroscience information framework: a data and knowledge environment for neuroscience. Neuroinformatics, 6, 149-160.

Glascher, J. (2009). Visualization of group inference data in functional neuroimaging. Neuroinformatics, 7, 73-82.

Goldberg, D. H., Victor, J. D., Gardner, E. P., \& Gardner, D. (2009). Spike train analysis toolkit: enabling wider application of information-theoretic techniques to neurophysiology. Neuroinformatics, 7, 165-178.

Gunay, C., Edgerton, J. R., Li, S., Sangrey, T., Prinz, A. A., \& Jaeger, D. (2009). Database analysis of simulated and recorded electrophysiological datasets with PANDORA's toolbox. Neuroinformatics, 7, 93-111.

Ichikawa, K. (2005). A modeling environment with three-dimensional morphology, A-Cell-3D, and $\mathrm{Ca}^{2+}$ dynamics in a spine. Neuroinformatics, 3, 49-64.

Kennedy, D. N. (2004). Barriers to the socialization of information. Neuroinformatics, 2, 367-368.

Koene, R. A., Tijms, B., van Hees, P., Postma, F., de Ridder, A., Ramakers, G. J., et al. (2009). NETMORPH: a framework for the stochastic generation of large scale neuronal networks with realistic neuron morphologies. Neuroinformatics, 7, 195-210.

Liu, T., Li, G., Nie, J., Tarokh, A., Zhou, X., Guo, L., et al. (2008). An automated method for cell detection in zebrafish. Neuroinformatics, 6, 5-21.

Luo, X. Z., Kennedy, D. N., \& Cohen, Z. (2009). Neuroimaging informatics tools and resources clearinghouse (NITRC) resource announcement. Neuroinformatics, 7, 55-56.

Nowinski, W. L., Volkau, I., Marchenko, Y., Thirunavuukarasuu, A., Ng, T. T., \& Runge, V. M. (2009). A 3D model of human cerebrovasculature derived from $3 \mathrm{~T}$ magnetic resonance angiography. Neuroinformatics, 7, 23-36.

Srinivasan, R., Zhou, X., Miller, E., Lu, J., Lichtman, J., \& Wong, S. T. (2007). Automated axon tracking of $3 \mathrm{D}$ confocal laser scanning microscopy images using guided probabilistic region merging. Neuroinformatics, 5, 189-203.

Versace, M., Ames, H., Leveille, J., Fortenberry, B., \& Gorchetchnikov, A. (2008). KInNeSS: a modular framework for computational neuroscience. Neuroinformatics, 6, 291-309.

Zhang, J., Liang, L., Anderson, J. R., Gatewood, L., Rottenberg, D. A., \& Strother, S. C. (2008). A Java-based fMRI processing pipeline evaluation system for assessment of univariate general linear model and multivariate canonical variate analysis-based pipelines. Neuroinformatics, 6, 123-134. 